\title{
In silico Analysis of Novel Mutation Ala102Pro targeting pncA Gene of $M$. tuberculosis
}

Dhanurekha Lakshmipathy, Gayathri Ramasubban, Lily Therese*, Umashankar Vetrivel, Muthukumaran Sivashanmugam, Sunitha Rajendiran, Sridhar R, Madhavan HN, and Meenakshi N

Vision Research Foundation, Chennai, Tamil Nadu, India

\begin{abstract}
This study reports on the structural and functional basis of pyrazinamide (PZA) resistance conferred by a novel mutation Ala102Pro in pncA gene as sequenced from a PZA resistant Mycobacterium tuberculosis strain. Molecular modeling studies of Wild Type (WT) and Mutant Type (MT) of Pyrazinamidase (PZase) showed the mutation at Ala102Pro does not impact on the conformation of the protein. However, the docking studies infer that MT has a higher inhibitory constant (Ki-990.0m) compared to WT (Ki-822.42m), which is indicative of drug resistance in MT. Furthermore, molecular interaction studies also reveal that WT forms 4 hydrogen bonds involved in PZA-WT inhibitory interactions, whereas, in case of MT, it formed 5 hydrogen bonds with PZA. However, Ala102 in WT was found to be less fluctuating and more stable in molecular dynamics simulation when compared to Pro102 in MT which was highly fluctuating and unstable. This implies that Ala102 shall be a key residue involved in PZA inhibitory interactions. Moreover, MT does not show hydrogen bonding with PZA with Pro102 and also deviating in terms of PZA binding pose in comparison with WT. Hence, the observed deviations in terms of MT-PZA interactions shall be attributed to the drug resistance conferred.
\end{abstract}

Keywords: Pyrazinamide; M. Tuberculosis; pncA gene

\section{Introduction}

Pyrazinamide (PZA) is the first-line drug used in the treatment of tuberculosis along with isoniazid and rifampicin and it inhibits semidormant mycobacteria only at low $\mathrm{pH}$ in vitro.

The $p n c A$ gene encodes pyraziniamidase (PZase), and mutations in $p n c A$ are associated with resistance to PZA $[1,2]$ or loss of PZase activity. PZA acts by targeting the fatty acid synthase/synthetase enzyme, and is responsible for the killing of persistent tubercle bacilli in the initial intensive phase of chemotherapy. It is a prodrug that is converted to its active form namely, pyrazinoic acid (POA) by the catalytic action of PZase enzyme, encoded by the $p n c A$ gene in $M$. tuberculosis. Interestingly, PZA is active only at low $\mathrm{pH}$ since acidic environment favours accumulation of POA in the cytoplasm due to an ineffective efflux pump, thereby leading to improper efflux out of the amidase from the cell to the exterior $[2,3]$.

Although a large number of mutations have been described, no mutational hotspots have been identified so far [4]. This can be explained by the fact that mutations occur along the entire length of the pncA gene [5]. However, studies show that mutations that confirm the PZA resistance occur mainly in the putative promoter region of the pncA gene.

Scorpio and Zhang in 1996 [6] had identified the PZase gene ( $p n c A$ ) from $M$. tuberculosis and had shown that $p n c A$ mutations are a major mechanism of PZA resistance [6]. The identified $p n c A$ mutations are largely missense mutations causing amino acid substitutions, and in some cases nucleotide insertions or deletions and nonsense mutations in the $p n c A$ structural gene or in the putative promoter region of $p n c A$ [7]. The uniqueness of the mutations of $p n c A$ gene is its diversity and scattering along the whole gene though there does appear to be some degree of clustering at three regions of $p n c A$ protein ( 3 to 17,61 to 85 , and 132 to 142). These regions are likely to contain catalytic sites for the Pzase enzyme [8]. The catalytic residues comprise the active site (D8, K96, A134 and C138) and the metal-binding site (D49, H51 and H71) $[9,10]$. Cys-138, Ala-134, Thr-135, Trp-68, and Asp-8 in the M. tuberculosis PZase could be key residues for hydrolysis of PZA [8]. At the protein level, these regions were found to be well conserved among the amino acid sequences of $p n c A$ proteins from different bacterial species.

The crystal structure of the M. tuberculosis pncA protein has been determined, showing significant differences in the substrate binding cavity when compared to the pyrazinamidases from Pyrococcus horikoshii and Acinetobacter baumanii. In M. tuberculosis, this region was found to hold a $\mathrm{Fe}^{2+}$ ion coordinated by one aspartate and three histidines, the most crucial structural element in this loop appears to be the specific positioning of residue His57 which is directly involved in the coordination of the $\mathrm{Fe}^{2+}$ ion. The overall architecture of the pyrazinamidase of $M$. tuberculosis is similar to that reported for the other pyrazinamidases of $A$. baumanii and P. horikoshii [11].

In the $p n c A$ model, the putative catalytic centre would be located in a pocket formed by one $\alpha$-helix ( $\alpha \mathrm{E})$, four $\beta$-strands ( $\beta 1, \beta 2, \beta 3$ and $\beta 4$ ) consisting of $\beta 1$ (Asp-8 and Phe-13), $\beta 2$ (Asp-49), $\beta 3$ (Lys-96), $\beta 4$ (Ala-134 and Thr-135) and $\alpha \mathrm{E}$ (Cys-138). In this pocket, the conserved active cysteine residue Cys-138 is located close to the conserved residues: Asp-8, Trp-68, Lys-96, Ser-104, Ala-134 and Thr-135. In the pncA model, the side chains of the two residues Asp- 8 and Lys-96 are found to point towards Cys-138 of the active-site. The modification of the amino acid residues Asp-8, Lys-96 and Ser-104 in the mutants D8G, K96T and S104R resulted in enzymes showing specific activities drastically impaired (\%0.004 unit $\mathrm{mg}$ ), thus suggesting that these

*Corresponding author: Dr. K. Lily Therese, Vision Research Foundation, Chennai, Tamil Nadu, India, E-mail: drklt@snmail.org

Received January 12, 2013; Accepted April 13, 2013; Published April 17, 2013

Citation: Lakshmipathy D, Ramasubban G, Therese L, Vetrivel U, Sivashanmugam M, et al. (2013) In silico Analysis of Novel Mutation Ala102Pro targeting pncA Gene of M. tuberculosis. J Comput Sci Syst Biol 6: 083-087. doi:10.4172/jcsb.1000103

Copyright: (c) 2013 Lakshmipathy D, et al. This is an open-access article distributed under the terms of the Creative Commons Attribution License,which permits unrestricted use, distribution, and reproduction in any medium, provided the original author and source are credited. 
residues are essential for the $p n c A$ activity. The amino acids found at positions $8,13,61,69,96,103,104$ and 146 are functionally and or structurally important in $p n c A$ [9]. Hence, in this study we attempt to utilize this empirical structural data to analyze to the impact of Ala102Pro mutation on PZA resistance.

\section{Materials and Methods}

\section{Phenotypic drug susceptibility testing}

Phenotypic PZA $(100 \mu \mathrm{g} / \mathrm{ml})$ susceptibility testing was performed by BACTEC MicroMGIT culture system following manufacturer's instruction (Becton Dickinson, USA) [12].

\section{DNA extraction}

DNA was extracted from the M. tuberculosis isolate by boiling at $80^{\circ} \mathrm{C}$ for 10 minutes followed by centrifugation at $3,000 \mathrm{rpm} .5 \mu \mathrm{l}$ of supernatant was used as the template DNA.

\section{PCR targeting pncA gene}

The amplification reactions contained $200 \mu \mathrm{M}$ of each dNTPs [dATP, dTTP, dGTP, dCTP (Bangalore Genei)], $1 \mu \mathrm{M}$ of outer and inner set of primers, $1 \times$ buffer $[10 \mathrm{mM}$ Tris $-\mathrm{HCl}(\mathrm{pH} 8.3), 50 \mathrm{mM} \mathrm{KCl}, 1.5 \mathrm{mM}$ $\mathrm{MgCl}_{2}$ ], 3 units of Taq DNA polymerase enzyme and $5 \mu$ of template DNA.PCRtargeting $p n c A$ genewasperformed with thefollowing forward and reverse primers pncA1: 5'GGCGTCATGGACCCTATATC3' and pncA2: 5'CAACAGTTCATCCCGGTTC3' [13] with the thermal profile for 30 cycles of denaturation at $95^{\circ} \mathrm{C}$ for $1 \mathrm{~min}$, annealing at $60^{\circ} \mathrm{C}$ for $30 \mathrm{sec}$ and extension at $72^{\circ} \mathrm{C}$ for $1 \mathrm{~min}$ which yielded a $670 \mathrm{bp}$ product. For each PCR run, negative control and positive control were included in the study. The PCR results were considered valid only when the negative control was negative without amplicon and the positive control yielded specific band.

\section{Detection of amplified product}

$10 \mu \mathrm{l}$ of the amplified product was subjected to electrophoresis on $2 \%$ agarose gel incorporated with $0.5 \mu \mathrm{g} / \mathrm{ml}$ ethidium bromide for visualization by UV transilluminator (Vilber Lourmet, France).

\section{Standard Precautions for PCR}

Strict and adequate precautions were taken to prevent amplicon getting contaminated. Separate rooms were used for the preparation of DNA, its amplification and analysis of amplified products. PCR cocktail was prepared in a laminar flow workbench; the microfuge tubes and milliQ water used for PCR were double sterilized.

\section{DNA sequencing and Data analysis}

DNA Sequencing of amplicons was carried out using an ABI prism 3110 automated DNA sequencer. Cycle sequencing of the amplified products was carried out using ABI Prism BigDye terminator kit (Applied Biosystems, USA) following manufacturer's instruction. The sequences were analyzed by Bio Edit sequence alignment software [14]. The sequences generated were compared with the wild type sequence (Genbank accession nos. for X68081 for katG) by using Multalin software [15] to identify the presence of mutation or polymorphism.

\section{Bioinformatics}

\section{Data sets}

The protein sequence for Pyrazinamidase (Pzase) pncA (UniProtKB id: Q50575) was obtained from UniProtKb to perform in silico sequence analysis. The 3D atomic coordinates (PDB ID:3PL1) were obtained from Protein Data Bank (http://www.pdb.org/pdb/home/home.do) for structure analysis, mutation modeling (Ala102Pro), molecular docking and molecular dynamics simulation studies.

Predicting stability change on single amino acid polymorphism based on support vector machine (I-Mutant 2.0)

Protein structural stability of the mutants were assessed using I-Mutant 2.0 server which is a support vector machine (SVM) - based tool for automatic prediction of protein stability changes upon singlepoint mutations; predictions are performed for both sequence and structure of proteins using I-Mutant 2.0 server. The output of this program displays the predicted free energy change value $(\Delta \Delta \mathrm{G})$ which is calculated from the unfolding Gibbs free energy value of the native type $(\mathrm{kcal} / \mathrm{mol})$. Positive $\Delta \Delta \mathrm{G}$ values infers that the mutated protein possesses high stability and vice versa (http://gpcr.biocomp.unibo.it/ cgi/predictors/ I-Mutant 2.0/ I-Mutant 2.0.cgi).

\section{Analysis of secondary structure using Protein Structure Prediction Server (PSIPRED v3.0)}

The PSIPRED is a reliable sequence based Protein Secondary Structure Prediction Server and was used in this study to predict the secondary structure of the mutant sequence so as to assess the impact of the novel mutation at the secondary structure elements. The output gives the details of presence of helix $(\mathrm{H})$, sheets $(\mathrm{E})$ and coils $(\mathrm{C})$ in the protein sequence with graphical representation (http://bioinf.cs.ucl. ac.uk/psipred/).

\section{Detection of conserved amino acids in the human pathogenic organisms containing pyrazinamidase/ nicotinamidase}

Consurf analysis can be utilized to reveal the conservation of residues among the group of organisms with common metabolic functions. Here, in this study, the bacteria which exhibit PZase activity were grouped together in terms of sequence identity and were further analyzed for conserved residues through consurf analysis.

\section{Homology modeling of mutant Pzase}

Homology modeling helps in predicting the 3D structure of a protein with unknown structure by comparing it with a known structure sharing high sequence similarity. In this study, MODELLER9v7 [16] was used for modeling the novel mutant protein. Since the crystal structure of the pyrazinamidase M. tuberculosis (PDB id: 3PL1) was available [12], the same was used as template to predict the mutant form. Finally, the stereochemcial property of WT and MT of the protein structure was predicted using SAVS server (http://nihserver. mbi.ucla.edu/SAVES/).

\section{Structure optimization and validation}

The WT and the modeled MT PZAse structures were subjected to energy minimization by steepest descent algorithm using GROMOS96 force field [17]. The quality of the protein structure modeled was again checked using Q-Mean server [18] and ProQ (http://www.sbc. su.se/ bjornw/ProQ/ProQ.cgi)

\section{Active site analysis of Pzase enzyme}

The active site of Pzase from M. tuberculosis was analyzed using Q-SiteFinder, CASTp (http://sts-fw.bioengr.uic.edu/castp/calculation. php) and Pocket Finder server (http://www.modelling.leeds.ac.uk/ 
Citation: Lakshmipathy D, Ramasubban G, Therese L, Vetrivel U, Sivashanmugam M, et al. (2013) In silico Analysis of Novel Mutation Ala102Pro targeting pncA Gene of M. tuberculosis. J Comput Sci Syst Biol 6: 083-087. doi:10.4172/jcsb.1000103

pocketfinder/help.html) to identify the binding site for docking studies. The results obtained were correlated with the active site residues from published literature $[7,8,19]$.

\section{Ligand optimization}

The initial structure of PZA was obtained from PubChem in 3D SDF format. It was converted to PDB format using Open Babel (http://www.molecularnetworks.com/online_demos/corina_demo. html). Hydrogen bonds were added to the structure and was geometry optimized (Broyden- Fletcher-Golfarb-Shanno line search method set to 1000 steps) using ArgusLab [20], wherein, the energy minimization was carried out using Universal Force Field (UFF) [21].

\section{Docking of PZA-Pzase}

PZA was docked into the structures of WT and MT using AutoDock 4.0 [22]. In this docking simulation, semiflexible docking protocols [23] were used, in which the protein structures were kept rigid and the PZA being docked was kept flexible. Blind docking was performed using grid point value (X, Y and Z) of $100 \AA ̊$ and spacing between the gird points was $0.375 \AA$. Lamarckian genetic algorithm was selected for ligand conformational searching and default docking parameters were used. The best docked complexes based on the lowest binding energy were further analyzed for hydrogen bonding interactions and the binding energy of WT and MT was compared. Finally, the Proteinligand complexes were analyzed using Discovery Studio Visualizer [24] and PYMOL visualization tool (http://www.pymol.org/).

\section{Docking complex simulation}

To study the stability of PZA ligand with wild and mutant forms, molecular dynamics simulation was carried out for the docked Proteinligand complexes. All simulations were performed using GROMACS [19], with GROMOS96 43al force field. The topology file for the ligand was generated using Dundee PRODRG Server [http://davapc1.bioch. dundee.ac.uk/prodrg/].

The system was placed at the center of a cubic periodic box with an area of $37 \times 37 \times 37 \AA$ and was solvated with a simple point charge (SPC213) water model. The distance between solute and edge of the box was $0.9 \AA$ for the system. It included water molecules for all the complexes. In order to neutralize the systems charge to zero, adequate number of $\mathrm{Na}^{+}$or $\mathrm{Cl}^{-}$ions were added. Each protein-ligand complex was subjected 2000 steps of energy minimization using steepest descent algorithm. Equilibration of the ensembles was performed by position restraint of the system for 100 ps. A constant temperature of $300 \mathrm{~K}$ and $1 \mathrm{~atm}$ pressure was maintained in the system. For long range electrostatics calculation, Partial Mesh Ewald coulomb type was used with a cut off of $1.0 \mathrm{~nm}$. All bonds including $\mathrm{H}$-bonds were constrained using LINCS algorithm. The MD simulation was carried out for 1 ns for each complex. After simulation, the trajectories were analyzed by ngmx software package. All the molecular modeling and simulation studies were carried on OPEN Discovery 2 Linux Platform [25].

\section{Results and Discussion}

\section{Phenotypic drug susceptibility testing}

Phenotypic drug susceptibility testing by BACTEC method showed the M. tuberculosis isolate to be resistant to PZA.

\section{PCR targeting the pncA gene and DNA sequencing}

PCR targeting the pncA gene resulted in a 670-bp amplified product. DNA sequencing of the amplified product with forward and reverse primers followed by MultAlin analysis of the M. tuberculosis strain revealed the presence of novel substitution mutation Ala102Pro (GCG $\rightarrow$ CCG).

\section{SNP Analysis of I-Mutant Server}

In this study, wild type sequence of $p n c A$ at $102^{\text {nd }}$ position Ala was replaced by Pro to predict protein stability changes through I-mutant server. The results infer loss of stability by the mutant protein with negative Gibbs free energy value of -1.10 at $\mathrm{pH} 5.5$ and $37^{\circ} \mathrm{C}$.

\section{Secondary structure analysis for Wild and Mutant Type of PZA (Pdbsum SERVER)}

PSIPred results for the MT shows no significant change when compared to WT and MT (Figure1A and figure 1B).

\section{ConSurf analysis}

Consurf analysis revealed the following residues to be conserved across all the selected Pzase activity exhibiting pathogenic organisms: Asp8, Phe13, Val44, Asp49, His51, His71, Gly78, Lys96, Tyr103, Thr114, Leu120, Gly132, Ala134, Asp136, Cys138, Val139, Ala146, Val155, Ala171, and Met175 and shall contribute to structural and functional aspects. As the novel mutation Ala102Pro was predicted to occur near the Tyr103 conserved region, it shall also contribute in the modulation of activity of the protein. Moreover, proline insertion shall also confer structural deviation as it forms partial peptide bond.
Figure 1a

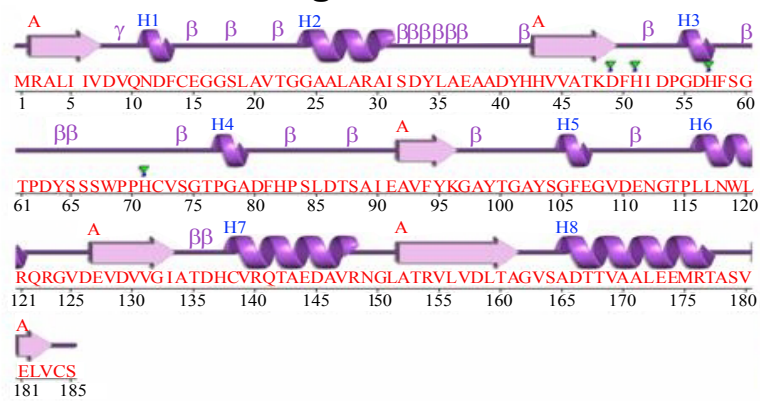

\section{Figure 1b}

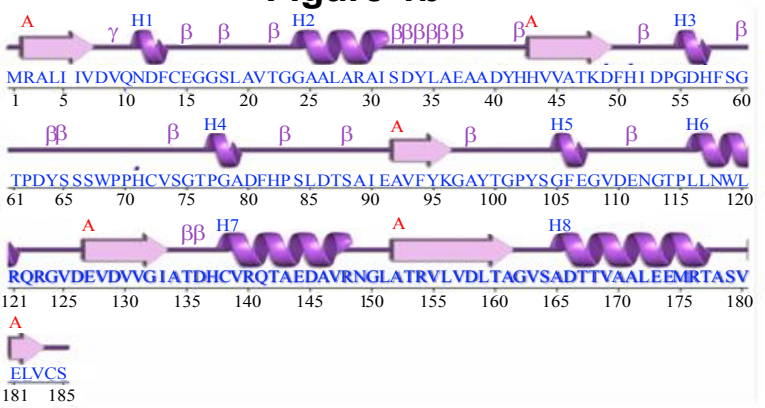

Figure 1: Secondary structure prediction of wild (1A) and mutation (1B) of PZA using pdbsum server. 
Citation: Lakshmipathy D, Ramasubban G, Therese L, Vetrivel U, Sivashanmugam M, et al. (2013) In silico Analysis of Novel Mutation Ala102Pro targeting pncA Gene of M. tuberculosis. J Comput Sci Syst Biol 6: 083-087. doi:10.4172/jcsb.1000103

\section{Homology modeling and loop refinement of MT Pzase}

The structure of MT Pzase (Ala102Pro) was modeled using Modeler9v7 with WT structure as template. The modeled structure was found to be highly plausible as it had $99 \%$ sequence identity with that of the template. Moreover, the Ramachandran plot also showed $83.5 \%$ of residues in most favored regions with no residues in disallowed region (Figure 2).

\section{Structure optimization and Validation}

The structures of WT and MT were energy minimized using GROMOS96 force field by Gromacs software package. The potential energy of WT and MT were found to be $-2.6391059 \mathrm{e}+04 \mathrm{Kcal} / \mathrm{mol}$ and MT $-2.3403371 \mathrm{e}+04 \mathrm{Kcal} / \mathrm{mol}$, respectively. The optimized structure was validated using Q-mean and ProQ Server (Table 1 and figure 3).

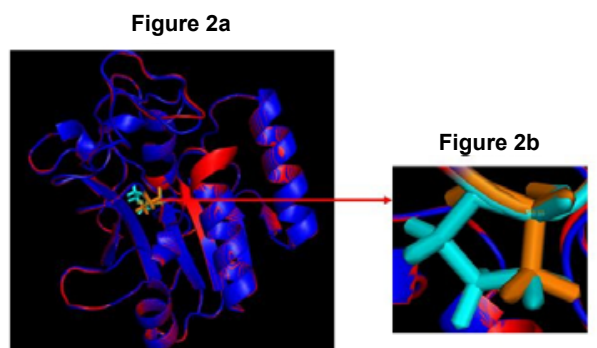

Figure 2: Superimposition of WT and MT PZase 3D structure. 2a) WT is coloured in blue and mutant in red. The Residue at position 102 is shown stickl form. 2b) Enlarged view at 102nd position (WT is colored in orange and MT in cyan).

\begin{tabular}{|l|l|l|l|l|}
\hline \multirow{2}{*}{$\begin{array}{l}\text { SAVS (Structure Analysis and } \\
\text { Validation Server) }\end{array}$} & \multicolumn{2}{|r|}{$\begin{array}{r}\text { Before Energy } \\
\text { Minimization }\end{array}$} & $\begin{array}{r}\text { After Energy } \\
\text { Minimization }\end{array}$ \\
\cline { 3 - 6 } & WILD & MUTANT & WILD & MUTANT \\
\hline Most favoured regions & $84.3 \%$ & $90.5 \%$ & $87.3 \%$ & $91.9 \%$ \\
\hline Additional allowed regions & $12.6 \%$ & $7.0 \%$ & $12.7 \%$ & $8.1 \%$ \\
\hline Generously allowed regions & $1.3 \%$ & $1.9 \%$ & $0 \%$ & $0 \%$ \\
\hline Disallowed regions & $1.9 \%$ & $0 \%$ & $0 \%$ & $0 \%$ \\
\hline ProQ LG score & 5.167 & 5.340 & 6.064 & 5.006 \\
\hline Max sub & 0.187 & 0.228 & 0.594 & 0.511 \\
\hline Q mean score & - & - & 0.82 & 0.79 \\
\hline
\end{tabular}

Table 1: Energy minimization and Protein structure Quality assessment of WT and MT of Pzase.

Figure 3a

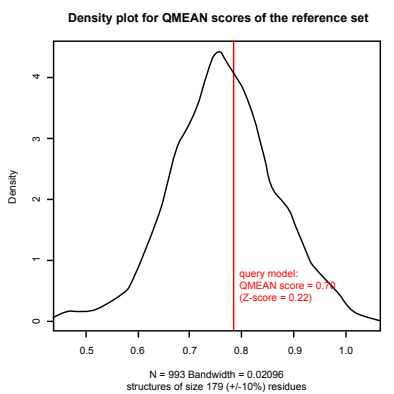

$N=993$ Bandwith $=0.02096$

Figure 3: Q-Mean Score of WT and MT of PZase.

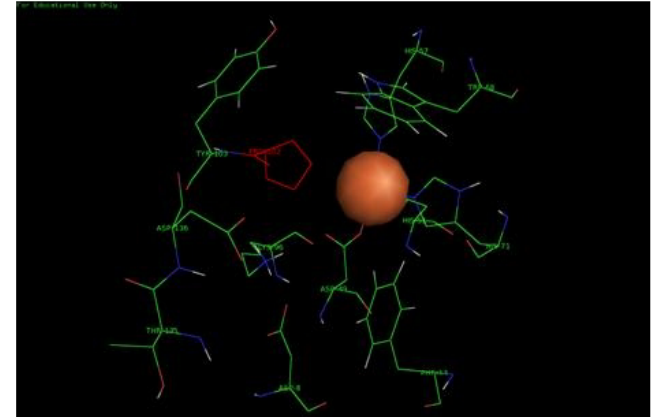

Figure 4: CASTp pocket prediction: Heme is indicated as brown sphere shape and Ala 102 Pro mutant position in Red and other pocket residues are indicated as lines with labels. Ala102 was found to structurally orient near the active site along with other heme binding residues: ASP49, HIS51, HIS57, HIS71. Hence the mutation in Ala102Pro shall affect the PZA activity in terms of substrate binding.

\begin{tabular}{|l|l|l|l|}
\hline Type & Binding energy & Ki value: $\boldsymbol{\mu m}$ & Hydrogen bond \\
\hline Wild & -4.21 & 822.42 & Ala102, Lys96, Asp8,Cys138 \\
\hline $\begin{array}{l}\text { Mutant } \\
\text { (Ala102Pro) }\end{array}$ & -4.1 & 990.0 & $\begin{array}{l}\text { Asp8, Asp49, cys138, Ala134, } \\
\text { Ile133 }\end{array}$ \\
\hline
\end{tabular}

\section{Active site analysis of PZA}

Three different methods namely, CASTp, Q-site and pocket finder were implemented to predict the active site residues of PZase. All the predictions were found to be synonymous and representing the same amino acid residues: Asp8, Phe13, Leu19, Asp49, His57, Trp68, His71, Lys96, Ala102, Tyr103, Thr135, Asp136, and Cys138 (Figure $4 \mathrm{a})$. Moreover, the predicted residues also correlated well with the documented studies. $[8,9,19]$.

\section{Docking studies of WT \&MT type of Pzase with PZA}

The docking studies were performed using the Autodock software. The aminoacid residues Asp8, Phe13, Thr61, Pro69, Lys96, Tyr103, and Cys 138 were assigned as catalytic region as these residues were proven to be potentially active site $[8,9,19]$.

The WT had binding energy of $-4.21 \mathrm{Kcal} / \mathrm{mol}$ and theoretical inhibitory constant (Ki) of $822.42 \mu \mathrm{M}$ (Table 2). The residues Asp8, Lys96, and Ala102 interacted through 4 hydrogen bonds with PZA. The interaction was found to span within the chosen active site $[8,9,19]$

The MT had binding energy of $-4.1 \mathrm{Kcal} / \mathrm{mol}$ and theoretical inhibitory (Ki) constant of $990.0 \mu \mathrm{M}$ (Table 2). The results of docking study infer that both WT and MT interact with PZA at the same binding pocket region. However, MT showed deviation in terms of interaction with PZA by excluding hydrogen bond formation with Lys96 and Ala 102 and including Ala134 and Ile133 (Figures 5A and 5B).

\section{Dynamics Simulation analysis}

To further validate the molecular docking studies, high performance molecular dynamics simulation protein-ligand complex were performed and from the resultant trajectory, root mean square fluctuation (RMSF) and gyration analysis were performed. The RMSF results showed that the MT has high fluctuation when compared to WT due to the acquired mutation (Figure 6A). Further, the Radius of gyration analysis also showed MT to be slightly compact than WT which shall interfere with the flexibility of the protein (Figure 6B). 
Citation: Lakshmipathy D, Ramasubban G, Therese L, Vetrivel U, Sivashanmugam M, et al. (2013) In silico Analysis of Novel Mutation Ala102Pro targeting pncA Gene of M. tuberculosis. J Comput Sci Syst Biol 6: 083-087. doi:10.4172/jcsb.1000103

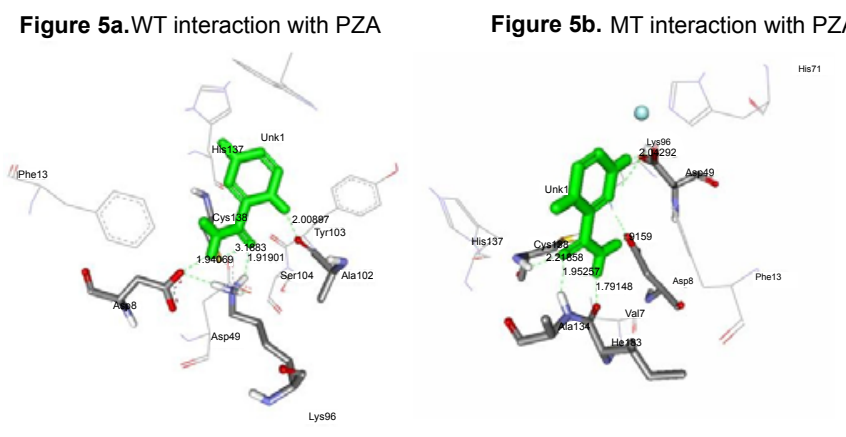

Figure 5: Docking Interaction of WT and MT PZase with PZA. PZA represented in Green colour.
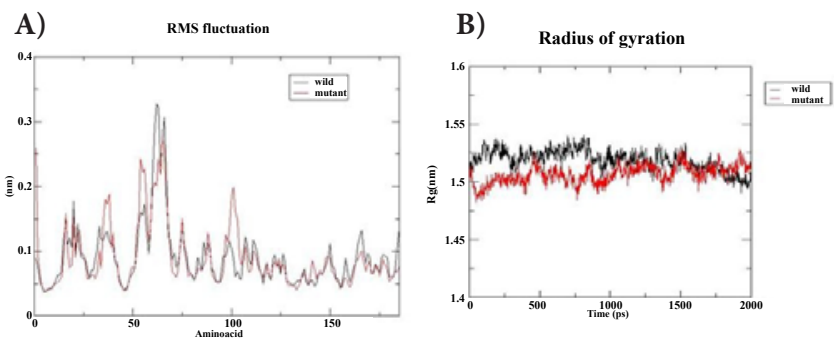

Figure 6: A) Represent Root Mean Square Fluctuation of residues in WT and MT type of PZase. B) Indicates the radius of gyration in WT \& MT type PZase. In both figures black lines indicate WT and Red lines for MT.

\section{Conclusion}

The novel mutation Ala102Pro was found to span in the putative active site (Lys96-Tyr103) region of PZase. The SNP analysis by I-mutant server showed that the stability of the protein was affected with negative Gibbs free energy value- 1.10 at $\mathrm{pH} 5.5$ and $37^{\circ} \mathrm{C}$. Molecular modeling and docking analysis showed that MT has a higher inhibitory constant than WT in terms of PZA binding, which indicates that the drug affinity is highly affected in the MT due to structural changes. The molecular dynamics simulation and gyration analysis together infer the change in flexibility at the active site cavity of MT. Hence, this study gives insight on the impact of novel mutation on the activity of this protein which can be attributed to the drug resistance observed. This study also presents a need for the discovery of potential newer drug molecules against both mutant and wild types.

\section{Acknowledgement}

The authors gratefully acknowledge the financial support by Chennai Willingdon Corporate Foundation (Chennai, India) for funding the research project.

\section{References}

1. Dalmer O, Walter E (1936) Verfahren zur Herstellung von Derivaten der Pyrazinmonocarbonsaure. DRP 623: 257.

2. Yeager RL, Munroe WG, Dessau FI (1952) Pyrazinamide (aldinamide) in the treatment of pulmonary tuberculosis. Am Rev Tuberc 65: 523-546.

3. Morlock GP, Crawford JT, Butler WR, Brim SE, Sikes D, et al. (2000) Phenotypic characterization of pncA mutants of Mycobacterium tuberculosis. Antimicrob Agents Chemother 44: 2291-2295.

4. Scorpio A, Lindholm-Levy P, Heifets L, Gilman R, Siddiqi S, et al. (1997) Characterization of pncA mutations in pyrazinamide-resistant Mycobacterium tuberculosis. Antimicrob Agents Chemother 41: 540-543.

5. Somoskovi A, Parsons LM, Salfinger M (2001) The molecular basis of resistance to isoniazid, rifampin, and pyrazinamide in Mycobacterium tuberculosis. Respir Res 2: 164-168.
6. Scorpio A, Zhang Y (1996) Mutations in pncA, a gene encoding pyrazinamidase/ nicotinamidase, cause resistance to the antituberculous drug pyrazinamide in tubercle bacillus. Nat Med 2: 662-667.

7. Sreevatsan S, Pan X, Zhang Y, Kreiswirth BN, Musser JM (1997) Mutations associated with pyrazinamide resistance in pncA of Mycobacterium tuberculosis complex organisms. Antimicrob Agents Chemother 41: 636-640.

8. Lemaitre N, Sougakoff W, Truffot-Pernot C, Jarlier V (1999) Characterization of New Mutations in Pyrazinamide-Resistant Strains of Mycobacterium tuberculosis and Identification of Conserved Regions Important for the Catalytic Activity of the Pyrazinamidase pncA. Antimicrob Agents Chemother 43: 1761 1763.

9. Lemaitre N, Callebaut I, Frenois F, Jarlier V, Sougakoff W (2001) Study of the structure-activity relationships for the pyrazinamidase (PncA) from Mycobacterium tuberculosis. Biochem J 353: 453-458.

10. Ramaswamy S, Musser JM (1998) Molecular genetic basis of antimicrobia agent resistance in Mycobacterium tuberculosis: 1998 update. Tuber Lung Dis 79: 3-29.

11. Petrella S, Gelus-Ziental N, Maudry A, Laurans C, Boudjelloul R, et al. (2011) Crystal structure of the pyrazinamidase of Mycobacterium tuberculosis: insights into natural and acquired resistance to pyrazinamide. PLoS One 6: e15785.

12. Salman H (2003) Siddiqui BACTEC 460TB System, Product and Procedure Manual MA0029, Becton Dickinson and Company, Maryland, USA.

13. Sekiguchi J, Miyoshi-Akiyama T, Augustynowicz-Kopeć E, Zwolska Z, Kirikae F, et al. (2007) Detection of Multidrug Resistance in Mycobacterium tuberculosis. J Clin Microbiol. 45: 179-192.

14. Hall TA (1999) BioEdit: a user-friendly biological sequence alignment edito and analysis program for Windows 95/98/NT. Nucleic Acids Symposium Series 41: 95-98.

15. Corpet F (1988) Multiple sequence alignment with hierarchical clustering Nucleic Acids Res 16: 10881-10890.

16. Eswar N, Webb B, Marti-Renom MA, Madhusudhan MS, Eramian D, et al (2007) Comparative protein structure modeling using MODELLER. Curr Protoc Protein Sci.

17. Hess B, Kutzner C, van der Spoel D, Lindahl E (2008) GROMACS 4: Algorithms for Highly Efficient, Load-Balanced, and Scalable Molecular Simulation. J Chem Theory Comp 4: 435-447.

18. Benkert P, Künzli M, Schwede T (2009) QMEAN server for protein mode quality estimation. Nucleic Acids Res 37: W510-514.

19. Du X, Wang W, Kim R, Yakota H, Nguyen H, et al. (2001) Crystal structure and mechanism of catalysis of a pyrazinamidase from Pyrococcus horikoshii. Biochemistry 40: 14166-14172.

20. Stoermer MJ (2006) Current status of virtual screening as analysed by target class. Med Chem 2: 89-112.

21. da Silva CH, Campo VL, Carvalho I, Taft CA (2006) Molecular modeling, docking and ADMET studies applied to the design of a novel hybrid for treatment of Alzheimer's disease. J Mol Graph Model 25: 169-175.

22. Huey R, Morris GM, Olson AJ, Goodsell DS (2007) A semiempirical free energy force field with charge-based desolvation. J Comput Chem 28:1145-1152.

23. Morris GM, Goodsell DS, Halliday RS, Huey R, Hart WE, et al. (1998) Automated docking using a Lamarckian genetic algorithm and an empirical binding free energy function. J Comput Chem 19:1639-1662.

24. Discovery Studio Modeling Environment (2011) Release 3.1, San Diego, Accelrys Software Inc.

25. Vetrivel U, Pilla K (2008) Open discovery: An integrated live Linux platform of Bioinformatics tools. Bioinformation 3: 144-146. 